Vol. $14 \mathrm{~N}^{\circ} 1$, pp.1-19, July 1995

Universidad Católica del Norte

Antofagasta - Chile

\title{
INTRODUCTION A LA THEORIE D'EQUISINGULARITE POUR LES FORMES DE PFAFF
}

\author{
ABDELHAK KABILA \\ Université de Valenciennes et du Hainaut Cambrésis, France
}

\section{Introduction.}

Dans ce travail, dont une version condensée et sans démonstrations a déjà été publiée au C.R.A.S. (cf. [5]), nous nous proposons d'une part d'établir un lemme d'isotopie pour les germes de formes de Pfaff intégrables, analogue dans un sens que nous préciserons ultérieurement au deuxième lemme d'isotopie de R. Thom pour les germes de fonctions; d'autre part, nous généralisons aux germes de formes de Pfaff la notion de nombre de J. Milnor d'un germe de fonction. Nous donnons ensuite le lien entre l'invariance de la multiplicité d'intersection, l'invariance du type topologique, la trivialité topologique et les conditions d'incidenses à la Whitney que nous allons définir ci-desous et qui généraliseront aux germes de formes de Pfaff la condition $\left(a_{f}\right)$ de $\mathrm{R}$. Thom et la condition (c) d'équisingularité de B. Teissier bien connues pour les germes de fonctions.

Plus précisément, écrivons $C^{n}=C^{2} \times C^{p}$, et désignons par:

$$
\omega(x, y, z)=a(x, y, z) d x+b(x, y, z) d y+\sum_{k=1}^{p} \quad c_{k}(x, y, z) d z_{k}
$$

un germe de formes de Pfaff holomorphes intégrables à l'origine 0 de $C^{n}$ où $(x, y) \in C^{2}$ et $z=\left(z_{1}, z_{2}, \cdots, z_{p}\right) \in C^{p} ; a, b, c_{1}, c_{2}, \cdots, c_{p}$ sont des éléments de l'anneau $\mathcal{O}_{C^{n}, 0}$ des germes de fonctions holomorphes à l'origine de $C^{n}$, la condition d'intégrabilité se traduisant par $\omega \wedge d \omega=0$. Notons $S$ le lieu singulier de $\omega$ c'est- $\grave{a}$-dire le germe d'ensembles analytique d'équation:

$$
S: a=b=c_{1}=c_{2}=\cdots=c_{p}=0 .
$$


Soit $U$ un voisinage ouvert de 0 dans $C^{n}$ sur lequel $\omega$ est holomorphe. Nous désignons par $\mathcal{F} \omega \mid U$ le germe de feuilletages holomorphes défini par $\omega$ sur $U-S$, et par $\mathcal{F} \omega=(S, \mathcal{F} \omega \mid U)$ le germe de feuilletages singuliers défini par $\omega$.

Pour l'étude que nous nous proposons de faire, il suffit de se limiter au cas où la codimension de $S$ est égale à 2 . En fait, si la codimension de $S$ est supérieure ou égale à 3 , le théorème de $\mathrm{B}$. Malgrange [11] nous assure l'existence d'une intégrale première ordinaire, et le problème se ramène à celui des fonctions. De plus, il est facile de voir que si nous prenons le soin de choisir les composantes $a, b, c_{1}, \cdots, c_{p}$ de $\omega$ sans facteurs communs, ce qui ne change pas le germe de feuilletages, le cas codimension de $S$ égale à 1 ne pourra pas avoir lieu.

\section{Rappels de la situation dans le cas des fonctions.}

Avant d'étudier le cas des formes de Pfaff rappelons la situation pour les fonctions.

Définition 1.1. Soit $M$ une variété et $S$ un sous-ensemble de $M$. Une stratification de Whitney de $S$ est une partition $\zeta=\{X\}_{X \in \zeta}$ de $S$ en sous-variétés localement fermées vérifiant les conditions suivantes:

i) $\zeta$ est localement fini.

ii) si $X \in \zeta$ alors $\bar{X}=\bigcup_{\substack{Y \in \zeta \\ Y \cap X \neq \phi}} Y$

iii) les strates vérifient les conditions (a) et (b) de Whitney définies cidessous:

Si $X$ et $Y$ sont deux variétés disjointes de $\mathbf{R}^{\mathbf{n}}$ telles que $Y \subset \bar{X}$, et si $y \in Y$ :

- On dit que $(X, Y, y)$ vérifie (a) si pour toute suite $\left(x_{n}\right)$ de $X, x_{n} \longrightarrow$ $y \in Y$ telle que $\lim T_{x_{n}} X=\tau$ existe, alors $\tau \supset T_{y} Y$.

- On dit que $(X, Y, y)$ vérifie (b) si pour toute suite $x_{i}$ de $X, x_{i} \longrightarrow y$ et pour toute suite $y_{i}$ de $Y, y_{i} \longrightarrow y$ telles que $\ell=\lim \left[x_{i}, y_{i}\right]$ existe et $\Delta=\lim T_{x_{i}} Y$ existe alors $\ell \subset \Delta$. 
Exemple 1.1:

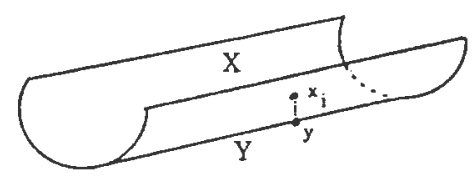

(a) vérifiée

Excmple 1.2:

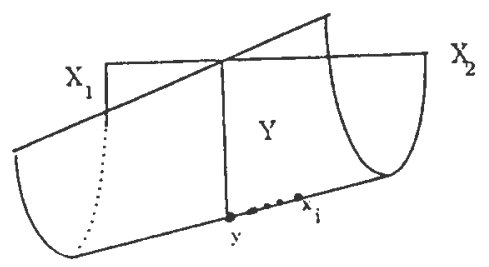

(i) 1 us verifice

\section{Exemple 1.3 :}

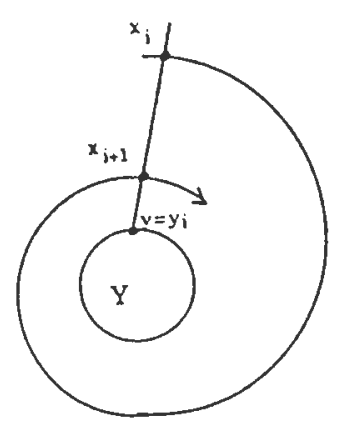
(a) verifiée
(b) non vérifiée

Remarque 1.1. L'importanc de ces conditions de régularité est bien connue parce qu'elles sont génériques et qu'elles ont des conséquences importantes dans la théorie de l'équisingularité des variétés analytiques complexes.

Définition 1.2. Soient $M$ et $M^{\prime}$ deux variétés $C^{\infty}$, et $f: M^{\prime} \longrightarrow M$ une fonction $C^{\infty}$. Désignons par $X$ et $Y$ deux sous-variétés de $M^{\prime}$ telles que $f_{\mid X}: X \longrightarrow M$ soit de rang constant. 
On dit que $f$ vérifie la condition $\left(a_{f}\right)$ de Thom pour $(X, Y)$ si pour toute suite $x_{i}$ de $X \quad x_{i} \longrightarrow y \in Y$ telle que $\tau=\lim _{i \rightarrow \infty} \operatorname{Ker} d_{x_{i}} f_{\mid X}$ existe alors $\tau \supset \operatorname{Ker}_{y} f_{\mid Y}$.

Définition 1.3. Soient $S$ (respectivement $S^{\prime}$ ) un fermé de $M$ (respectivement $M^{\prime}$ ) stratifié par $\zeta$ (respectivement par $\zeta^{\prime}$ ) et $f: M^{\prime} \longrightarrow M$ une fonction $C^{\infty}$ telle que $f\left(S^{\prime}\right) \subset S$.

On dit que $f$ est de Thom si:

i) $f_{\mid S^{\prime}}$ est propre.

ii) $\left(a_{f}\right)$ est vérifiée pour toute paire de strate $\left(X^{\prime}, Y^{\prime}\right)$ de $\zeta^{\prime}$.

iii) si $X^{\prime} \in \zeta^{\prime}$, il existe une strate $X$ de $\zeta$ telle que $f\left(X^{\prime}\right) \subset X$ et $f_{\mid X^{\prime}}: X^{\prime} \longrightarrow X$ soit une submersion.

Enoncé du 2ème lemme d'isotopie de Thom (cf [13]):

Soit $f: M^{\prime \prime} \longrightarrow M$ de Thom et $\pi: M \longrightarrow P$ tel que $\pi$ soit propre et $\pi_{\mid X}: X \longrightarrow P$ soit une submersion pour toute strate $X$.

Alors pour tout $p \in P$, il existe un ouvert $U(p)$ et des homéomorphismes $h$ et $h^{\prime}$ tels que le diagramme suivant soit commutatif:

$$
\begin{aligned}
& f^{-1} \pi^{-1} U(p) \underset{f}{\longrightarrow} \pi^{-1} U(p) \\
& \downarrow h^{\prime} \quad \downarrow h \\
& U(P) \times S_{p}^{\prime} \underset{1_{U(p)} \times f_{\mid S_{p}^{\prime}}}{\longrightarrow} U(p) \times S_{p}
\end{aligned}
$$

où $S_{p}=S \cap \pi^{-1}(p)$ et $S_{p}^{\prime}=S \cap f^{-1}\left(\pi^{-1}(p)\right)$. De plus $h$ et $h^{\prime}$ envoient strate sur strate et restreints à chaque strate ce sont des difféomorphismes.

\section{Généralisation aux formes de Pfaff intégrables.}

Avant de passer àl'extension des définitions aux formes de Pfaff intégrables rappelons que le deuxième lemme d'isotopie de $\mathrm{R}$. Thom dit donc grossomodo qu'une fonction holomorphe $f$ qui vérifie la condition $\left(a_{f}\right)$ de $\mathrm{R}$. Thom est topologiquement triviale. Or la condition $\left(a_{f}\right)$ porte uniquement sur $d f$ et non sur $f$, de même la notion de trivialité topologique porte uniquement sur le feuilletage défini par $f$ et non sur $f$ d'où les définitions suivantes:

Définition 2.1. Soient $\omega$ un germe de forme de Pfaff holomorphe in tégrable à l'origine 0 de $C^{n}, M$ une sous variété de $C^{n}$, et $m_{0}$ un point de $M$. 
Nous dirons que la condition ( $\left.a_{\omega}\right)$ est vérifiée relativement à $M$ au point $m_{0}$ si pour toute suite d'éléments de $C^{n}:\left(\alpha_{j}\right)_{j \in \mathbf{N}} ; \alpha_{j} \underset{j \rightarrow \infty}{\longrightarrow} m_{0} ; \alpha_{j} \notin M$, telle que $\lim _{j \rightarrow \infty} \operatorname{Ker} \omega\left(\alpha_{j}\right)=\Gamma$ existe, alors $\Gamma$ contient l'espace tangent $T_{m_{0}} M$ à $M$ au point $m_{0}$.

Nous dirons que la condition $\left(a_{\omega}\right)$ est vérifiée relativement à $M$ si elle est vérifiée relativement à $M$ en tout point de $M$.

Remarque 2.1. La condition $\left(a_{\omega}\right)$ est une généralisation aux formes différentielles de la condition $\left(a_{f}\right)$ de $\mathrm{R}$. Thom pour les germes de fonctions de $C^{n}$ dans $C$. Il est clair que dire qu'un germe de fonctions $f: C^{n} \longrightarrow C$ vérifie la condition $\left(a_{f}\right)$ de $R$.Thom signifie que le germe de formes de Pfaff $\omega=d f$ vérifie la condition $\left(a_{\omega}\right)$ défine ci-dessus.

Soit $\omega$ un germe de formes de Pfaff holomorphes intégrables à l'origine 0 de $C^{n}$ dont le lieu singulier $S$ est une sous-variété lisse de codimension 2, disons $S=\{(0,0)\} \times C^{p}$, et désignons par $S_{0}$ le lieu singulier de $\omega_{0}$ où $\omega_{0}$ est la restriction de $\omega$ à $C^{2} \times\left\{0_{C p}\right\}$.

Définition 2.2. Nous dirons que $\omega$ est topologiquement trivial au-dessus de $\omega_{0}$, s'il existe un germe d'homéomorphismes $\phi$ de $\left(C^{n} .0\right)$ tels que:

i) $\phi(S)=S_{0} \times C^{p}$.

ii) $\phi$ transforme le germe de feuilletages défini par $\omega$ en le produit par $C^{p}$ du germe de feuilletages défini $\omega_{0}$.

iii) $\phi$ commute avec la projection sur $C^{p}$.

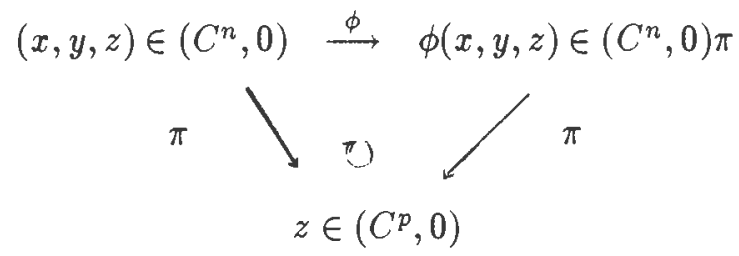

Une vraie généralisation aux formes de Pfaff intégrables du deuxième lemme d'isotopie de $\mathrm{R}$. Thom serait grossomodo: "si $\omega$ vérifie la condition $\left(a_{\omega}\right)$ alors $\omega$ est topologiquement trivial". Nous ne saurons démontrer ce résultat que pour une classe de germes de formes de Pfaff baptisées dans [4] "super-élémentaires" tels que les germes de formes de Pfaff logarithmiques. Elles ont la particularité de posséder un nombre fini de séparatrices et la propriété que toute feuille qui adhère analytiquement en 0 est analytique (cf [4]). Signalons d'ailleurs que l'hypothèse $\left(a_{\omega}\right)$ nous parait assez faible 
pour donner un tel résultat dans le cas général (cf démonstration du lemme d'isotopie dans le paragraphe suivant).

Pour pallier à ce fait nous avons posé les définitions suivantes :

Définition 2.3. (cf [10]). Soient $T_{1}$ et $T_{2}$ deux sous-espaces de $C^{n}$ considéré comme espace hermitien muni de la forme hermitienne:

$$
\left(\left(z_{i}\right)_{1 \leq i \leq n^{\prime}}\left(z_{j}^{\prime}\right)_{1 \leq j \leq n}\right) \stackrel{\longleftrightarrow}{\longrightarrow} \sum_{i=1}^{n} z_{i} \bar{z}_{i}^{\prime} .
$$

On suppose que: $\operatorname{dim}_{C} T_{1} \geq \operatorname{dim}_{C} T_{2} \geq 1$.

On appelle distance de $T_{1}$ à $T_{2}$ (dans cet ordre) et on note $d\left(T_{1}, T_{2}\right)$ la quantité:

$$
d\left(T_{1}, T_{2}\right)=\sup _{\substack{t_{2} \in T_{2} \\ t_{1} \in T_{1}^{\perp}-\{0\}}}\left\{\frac{\left|\left\langle t_{1}, t_{2}\right\rangle\right|}{\left\|t_{1}|| \cdot|| t_{2}\right\|}\right\}
$$

où $T_{1}^{\perp}$ désigne l'orthogonal de $T_{1}$ par rapport à la forme hermitienne $\langle$,$\rangle .$

Définition 2.4. Soient $\omega$ un germe de formes de Pfaff holomorphes intégrables à l'origine 0 de $C^{n}, M$ une sous-variété de $C^{n}$, et soit $m_{0}$ un point de $M$. Nous dirons que la condition $\left(c_{\omega}\right)$ est vérifiée relativement à $M$ au point $m_{0}$ s'il existe une constante $\lambda>0$, et un voisinage ouvert $V$ de $m_{0}$ dans $C^{n}$ tel que:

$$
d\left(\operatorname{Ker} \omega(v), T_{m_{0}} M\right) \leq \lambda d(v, M) \quad \text { pour tout } v \text { dans } V .
$$

Nous dirons que la condition $\left(c_{\omega}\right)$ est vérifiée relativement à $M$ si elle est vérifiée relativement à $M$ en tout point de $M$.

Remarque 2.2. La condition $\left(c_{\omega}\right)$ est une généralisation aux formes différentielles de la condition (C) de B. Teissier pour les germes de fonctions (cf. [10]). Il est clair que dire qu'un germe de fonctions $f: C^{n} \longrightarrow C$ vérifie la condition (C) de $\mathrm{B}$. Tessier signifie que le germe de formes de Pfaff $\omega=d f$ vérifie la condition $\left(c_{\omega}\right)$ définie ci-dessus. Rappelons aussi (Voir les travaux de B. Teissier [11] [12], de J. Briançon et J-P. Speder [1], [9] que la condition (c) équivaut á la condition (b) dans le cas des hypersurfaces.

Comme pour les fonctions, nous avons les propositions suivantes dont les démonstrations se trouvent en détail dans [4].

Proposition 2.1. Dire que la condition $\left(a_{\omega}\right)$ est vérifiée relativement à $\{(0,0)\} \times C^{p}$ signifie que pour tout $k, 1 \leq k \leq p: \frac{c_{k}}{\sqrt{|a|^{2}+|b|^{2}}}$ tend vers 0 le long de $\{(0,0)\} \times C^{p}$. 
Proposition 2.2. Dire que la condition $\left(c_{\omega}\right)$ est vérifiée relativement à $\{(0,0)\} \times C^{p}$ au point $\left(0,0, z^{0}\right) \in\{(0,0)\} \times C^{p}$ signifie qu'il existe une constante $\lambda>0$ et un voisinage ouvert $V$ de $\left(0,0, z^{0}\right)$ dans $C^{n}$ tels que pour tout $k, 1 \leq k \leq p$.

$$
\frac{\left|c_{k}(x, y, z)\right|}{\sqrt{|a(x, y, z)|^{2}+|b(x, y, z)|^{2}}} \leq \lambda \sqrt{|x|^{2}+|y|^{2}}
$$

pour tout $(x, y, z)$ dans $V$.

Remarque 2.3. Il est clair que $\left(c_{w}\right)$ implique $\left(a_{w}\right)$ mais la réciproque est moins évidente. Nous ne savons la démonstrer que pour certaines classes de formes de Pfaff telles que les formes de Pfaff super-élémentaires ou logarithmiques (cf. [4]).

Nous sommes maintenant en mesure d'énoncer le résultant principal de cet article.

Lemme d'isotopie. Soit $\omega$ un germe de formes de Pfaff holomorphes intégrables à l'origine 0 de $C^{n}$ dont le lieu singulier est une sous-variété lisse de codimension 2. Si la condition $\left(c_{\omega}\right)$ est vérifiée relativement à $S$ alors $\omega$ est topologiquement trivial.

Remarque 2.4. Le résultat ci-dessus reste vrai si nous replaçons le mot "homomorphe" par" $C^{\infty}$ " ou par "analytique réel".

Remarque 2.5. Dans le cas des fonctions i.e si $\omega=d f$ il est bien connu que la condition $\left(c_{\omega}\right)$ est équivalente à la condition $\left(a_{\omega}\right)$.

Démonstration du lemme d'isotopie. Elle fait partie du folklore et se fait à la main.

Ecrivons:

$$
\begin{gathered}
C^{n}=C^{2} \times C^{p} \\
\omega(x, y, z)=a(x, y, z) d x+b(x, y, z) d y+\sum_{j=1}^{p} c_{k}(x, y, z) d z_{k}
\end{gathered}
$$

avec $(x, y) \in C^{2}$ et $z=\left(z_{1}, z_{2}, \cdots, z_{p}\right) \in C^{p}$.

$$
\begin{gathered}
\omega_{0}(x, y)=a(x, y, 0) d x+b(x, y, 0) d y \\
x=x^{\prime}+i x^{\prime \prime}, \quad y=y^{\prime}+i y^{\prime \prime} \\
z_{k}=z_{k}^{\prime}+i z_{k}^{\prime \prime}, \quad 1 \leq k \leq p
\end{gathered}
$$

Nous construisons $2 p$ germes de champs de vecteurs réels $\chi_{1}^{\prime}, \chi_{2}^{\prime}, \cdots, \chi_{p}^{\prime}$, 
$\chi_{1}^{\prime \prime}, \chi_{2}^{\prime \prime}, \cdots, \chi_{p}^{\prime \prime}$ continus et tangents aux feuilles du feullitage $\mathcal{F}_{\omega}$ défini par $\omega$.

Les champs de vecteurs $\chi_{1}^{\prime}, \chi_{2}^{\prime}, \cdots, \chi_{p}^{\prime}, \chi_{1}^{\prime \prime}, \chi_{2}^{\prime \prime}, \cdots, \chi_{p}^{\prime \prime}$ sont définis comme suit:

$$
\begin{aligned}
& \chi_{k}^{\prime}=\left\{\begin{array}{l}
\frac{\partial}{\partial z_{k}^{\prime}} \text { sur } S . \\
A_{k}^{\prime} \frac{\partial}{\partial x^{\prime}}+A_{k}^{\prime \prime} \frac{\partial}{\partial x^{\prime \prime}}+B_{k}^{\prime} \frac{\partial}{\partial y^{\prime}}+B_{k}^{\prime \prime} \frac{\partial}{\partial y^{\prime \prime}}+\frac{\partial}{\partial z_{k}^{\prime}} \text { en dehors de } S .
\end{array}\right. \\
& \chi_{k}^{\prime \prime}=\left\{\begin{array}{l}
\frac{\partial}{\partial z_{k}^{\prime \prime}} \operatorname{sur} S . \\
-A_{k}^{\prime \prime} \frac{\partial}{\partial x^{\prime}}+A_{k}^{\prime} \frac{\partial}{\partial x^{\prime \prime}}-B_{k}^{\prime \prime} \frac{\partial}{\partial y^{\prime}}+B_{k}^{\prime} \frac{\partial}{\partial y^{\prime \prime}}+\frac{\partial}{\partial z_{k}^{\prime \prime}} \text { en dehors de } S .
\end{array}\right.
\end{aligned}
$$

pour $1 \leq k \leq p$ avec:

$$
\begin{aligned}
& A_{k}^{\prime}=\operatorname{Re} \frac{-\vec{a} c_{k}}{|a|^{2}+|b|^{2}}, A_{k}^{\prime \prime}=\operatorname{Im} \frac{-\bar{a} c_{k}}{|a|^{2}+|b|^{2}} \\
& B_{k}^{\prime}=\operatorname{Re} \frac{-\bar{b} c_{k}}{|a|^{2}+|b|^{2}}, B_{k}^{\prime \prime}=\operatorname{Im} \frac{-\bar{b} c_{k}}{|a|^{2}+|b|^{2}}
\end{aligned}
$$

Dans tout ce qui suit nous nous limitons à faire les calculs et les vérifications pour les champs de vecteurs $\chi_{k}^{\prime}$. Quant aux champs de vecteurs $\chi_{k}^{\prime \prime}$, le raisonnement est analogue. Il est clair que pour chaque $k$ compris entre 1 et $p$, le champ de vecteurs $\chi_{k}^{\prime}$ est de classe $C^{\infty}$ en dehors de $S$.

De plus pour $(x, y, z) \in U$ et $z_{0} \in C^{p}$ on a:

$$
\left\|\chi_{k}^{\prime}(x, y, z)-\chi_{k}^{\prime}\left(0,0, z_{0}\right)\right\|=\frac{\left|c_{k}(x, y, z)\right|}{\sqrt{|a(x, y, z)|^{2}+|b(x, y, z)|^{2}}}
$$

ce qui montre en vertu de la proposition (2.2) que le champ de vecteurs $\chi_{k}^{\prime}$ est lipschitzien le long de $\mathrm{S}$. Nous en déduisons qu'il est intégrable puisqu'il est de classe $C^{\infty}$ en dehors de $S$.

En écrivant:

$$
\omega=\omega^{\prime}+\omega^{\prime \prime}
$$

on vérifie sans peine que:

$$
\omega^{\prime}\left(\chi_{k}^{\prime}\right)=0 \text { et } \omega^{\prime \prime}\left(\chi_{k}^{\prime}\right)=0
$$

ce qui montre que le champ de vecteurs $\chi_{k}^{\prime}$ est tangent aux feuilles du feuilletage $\mathcal{F}_{\omega}$ défini par $\omega$. 
Appelons $\phi_{k, s}^{\prime}$ le groupe à un paramètre du champ de vecteurs $\chi_{k}^{\prime}$ et montrons qu'il existe un voisinage $W_{k}^{\prime}$, compact, de 0 dans $R^{4+2 p}$ et un nombre réel $\beta_{k}^{\prime}$ strictement positif tel que pour tout $m \in W_{k}^{\prime} \cap C^{2} \times\left\{0_{C^{p}}\right\}$ on ait:

$$
] s_{k,-}^{\prime}(m), s_{k,+}^{\prime}(m)[\text { contient }]-\beta_{k}^{\prime},+\beta_{k}^{\prime}[
$$

où $] s_{k,-}^{\prime}(m), s_{k,+}^{\prime}(m)$ [ désigne l'intervalle maximum sur lequel on peut intégrer le champ de vecteurs $\chi_{k}^{\prime}$ en partant du point $m$ à l'instant $s=0$ (avec condition initiale $m$ ).

La condition $\left(c_{\omega}\right)$ étant vérifiée relativement à $S$, il existe une constante $\lambda>0$ et un voisinage ouvert $V$ de 0 dans $C^{n}=C^{2} \times C^{p}$ tels que pour tout $k$ compris entre 1 et $p$ :

$$
\frac{\left|c_{k}(x, y, z)\right|}{\sqrt{|a(x, y, z)|^{2}+|b(x, y, z)|^{2}}} \leq \lambda \sqrt{|x|^{2}+|y|^{2}} \text { pour }(x, y, z) \text { dans } V .
$$

Prenons:

$$
\beta_{k}^{\prime}=1 / 2 \lambda
$$

$W_{k}^{\prime}=\overline{B(0, \rho) \cap V}$ l'adhérence de l'intersection de $V$ avec la boule de centre 0 et de rayon $\rho$, où $\rho$ est un réel strictement positif assez petit.

Soit $m_{0}=\left(x_{0}^{\prime}, x_{0}^{\prime \prime}, y_{0}^{\prime}, y_{0}^{\prime \prime}, O\right)$ un point de $W_{k}^{\prime} \in C^{2} \times\left\{O_{C^{p}}\right\}$ et vérifions que:

$$
] s_{k,-}^{\prime}\left(m_{0}\right), s_{k,+}^{\prime}\left(m_{0}\right)[\text { contient }]-1 / 2 \lambda,+1 / 2 \lambda[\text {. }
$$

Considérons l'équation différentielle $\left(E_{k}^{\prime}\right)$ suivante avec la condition initiale $m_{0}(\mathbf{0})=\mathbf{0}$.

$$
\left(E_{k}^{\prime}\right):\left\{\begin{array}{l}
\frac{d x^{\prime}}{d s}=A_{k}^{\prime}\left(x^{\prime}, x^{\prime \prime}, y^{\prime}, y^{\prime \prime}, z_{1}^{\prime}, z_{1}^{\prime \prime}, z_{2}^{\prime}, z_{2}^{\prime \prime}, \cdots, z_{p}^{\prime}, z_{p}^{\prime \prime}\right) \\
\frac{d x^{\prime \prime}}{d s}=A_{k}^{\prime \prime}\left(x^{\prime}, x^{\prime \prime}, y^{\prime}, y^{\prime \prime}, z_{1}^{\prime}, z_{1}^{\prime \prime}, z_{2}^{\prime}, z_{2}^{\prime \prime}, \cdots, z_{p}^{\prime}, z_{p}^{\prime \prime}\right) \\
\frac{d y^{\prime}}{d s}=B_{k}^{\prime}\left(x^{\prime}, x^{\prime \prime}, y^{\prime}, y^{\prime \prime}, z_{1}^{\prime}, z_{1}^{\prime \prime}, z_{2}^{\prime}, z_{2}^{\prime \prime}, \cdots, z_{p}^{\prime}, z_{p}^{\prime \prime}\right) \\
\frac{d y^{\prime \prime}}{d s}=B_{k}^{\prime \prime}\left(x^{\prime}, x^{\prime \prime}, y^{\prime}, y^{\prime \prime}, z_{1}^{\prime}, z_{1}^{\prime \prime}, z_{2}^{\prime}, z_{2}^{\prime \prime}, \cdots, z_{p}^{\prime}, z_{p}^{\prime \prime}\right) \\
\frac{d z_{k}^{\prime}}{d s}=1 \\
\frac{d z_{j}^{\prime}}{d s}=0 \text { pour } j \neq k, 1 \leq j \leq p \\
\frac{d z_{j}^{\prime}}{d s}=0 \text { pour } 1 \leq j \leq p
\end{array}\right.
$$


Cette équation différentielle se ramène à l'équation suivante:

$$
\left(E_{k}^{\prime \prime}\right):\left\{\begin{array}{l}
\frac{d x^{\prime}}{d s}=A_{k}^{\prime}\left(x^{\prime}(s), x^{\prime \prime}(s), y^{\prime}(s), y^{\prime \prime}(s), 0,0, \cdots, 0, s, 0, \cdots, 0,0\right) \\
\frac{d x^{\prime \prime}}{d s}=A_{k}^{\prime \prime}\left(x^{\prime}(s), x^{\prime \prime}(s), y^{\prime}(s), y^{\prime \prime}(s), 0,0, \cdots, 0, s, 0, \cdots, 0,0\right) \\
\frac{d y^{\prime}}{d s}=B_{k}^{\prime}\left(x^{\prime}(s), x^{\prime \prime}(s), y^{\prime}(s), y^{\prime \prime}(s), 0,0, \cdots, 0, s, 0, \cdots, 0,0\right) \\
\frac{d y^{\prime \prime}}{d s}=B_{k}^{\prime \prime}\left(x^{\prime}(s), x^{\prime \prime}(s), y^{\prime}(s), y^{\prime \prime}(s), 0,0, \cdots, 0, s, 0, \cdots, 0,0\right)
\end{array}\right.
$$

Démonstrons par exemple que:

$$
s_{k,+}^{\prime}\left(m_{0}\right)>+\frac{1}{2 \lambda} .
$$

Considérons l'ensemble $K$ suivant:

$$
K=\left\{m \in W_{k}^{\prime} \cap C^{2} \times\{0\} /\left\|m-m_{0}\right\| \leq \rho / 2\right\}
$$

$K$ est compact donc il existe $\varepsilon>0$ tel que pour tout $s$, $s_{k,+}^{\prime}\left(m_{0}\right)-\varepsilon<s \leq s_{k,+}^{\prime}\left(m_{0}\right)$ on ait:

$$
\left\|m(s)-m_{0}(0)\right\|>\rho / 2
$$

posons:

$$
\begin{gathered}
m(s)=\left(x^{\prime}(s), x^{\prime \prime}(s), y^{\prime}(s), y^{\prime \prime}(s)\right) . \\
\left\|m(s)-m_{0}(0)\right\|=\left\|\int_{0}^{s}\left(\frac{d m}{d u}\right) d u\right\| \leq \int_{0}^{s}\left\|\frac{d m}{d u}\right\| \cdot d u \\
=\int_{0}^{s} \sqrt{\left(A^{\prime}(m(u))^{2}+\left(A_{k}^{\prime \prime}(m(u))^{2}+\left(B_{k}^{\prime}(m(u))^{2}+\left(B_{k}^{\prime \prime}(m(u))^{2}\right)\right.\right.\right.} \cdot d u \\
=\int_{0}^{s} \frac{\left|c_{k}(m(u))\right|}{\sqrt{|a(m(u))|^{2}+|b(m(u))|^{2}}} \cdot d u \leq \int_{0}^{s} \lambda \cdot\|m(u)\| \cdot d u \\
=\int_{0}^{s} \lambda \rho d u=\lambda \cdot \rho \cdot s .
\end{gathered}
$$

Nous avons donc:

$$
\lambda \cdot \rho \cdot s \geq\left\|m(s)-m_{0}(0)\right\|>\rho / 2
$$

ce qui entraine: 


$$
s_{k,+}^{\prime}\left(m_{0}\right) \geq s>+\frac{1}{2 \lambda}
$$

d'où le résultat.

Un raisonnement analogue permet de montrer que

$$
s_{k,+}^{\prime}\left(m_{0}\right) \geq s<-\frac{1}{2 \lambda}
$$

d'où:

$$
] s_{k,-}^{\prime}\left(m_{0}\right), s_{k,+}^{\prime}\left(m_{0}\right)[\supset]-1 / 2 \lambda,+1 / 2 \lambda[
$$

Si nous appelons $\phi_{k, s}^{\prime \prime}$ le groupe à un parametre du champ de vecteur $\chi_{k}^{\prime \prime}$, pour $k$ compris entre 1 et $p$, nous pouvons établir de la meme façon l'existence d'un voisinage $W_{k}^{\prime \prime}$, compact, de 0 dans $\mathbf{R}^{4+2 p}$ et d'un nombre réel $\beta_{k}^{\prime \prime}$ strictement positif tel que pour tout $m \in W_{k}^{\prime \prime} \cap \mathbf{C}^{2} \times\left\{0_{\mathbf{C}^{p}}\right\}$ on ait:

$$
] s_{k,-}^{\prime \prime}(m), s_{k,+}^{\prime \prime}(m)[\text { contient }]-\beta_{k}^{\prime \prime},+\beta_{k}^{\prime \prime}[
$$

où $] s_{k,-}^{\prime \prime}(m), s_{k,+}^{\prime \prime}(m)$ [ désigne l'intervalle maximum sur lequel on peut intégrer le champ de vecteurs $\chi_{k}^{\prime \prime}$ en partant du point $m$ à l'instant $s=0$ (avec condition initiale $m$ ). Nous avons donc les applications continues suivantes:

$$
\begin{gathered}
\left.\stackrel{\circ}{W}_{k}^{\prime} \cap \mathbf{C}^{2} \times\left\{0_{\mathbf{C}_{p}}\right\} \times\right]-\beta_{k ;}^{\prime}+\beta_{k}^{\prime}\left[\stackrel{\phi_{k}^{\prime}}{\longrightarrow} \mathbf{R}^{4+2 p}\right. \\
\left(x^{\prime}, x^{\prime \prime}, y^{\prime}, y^{\prime \prime}, 0, s_{k}^{\prime}\right) \longmapsto \emptyset_{k}^{\prime}\left(x^{\prime}, x^{\prime \prime}, y^{\prime}, y^{\prime \prime}, 0, s_{k}^{\prime}\right)=\phi_{k, s_{k}^{\prime} k}^{\prime}\left(x^{\prime}, x^{\prime \prime}, y^{\prime}, y^{\prime \prime}, 0\right) \\
\left.\stackrel{\circ}{W}_{k}^{\prime \prime} \cap \mathbf{C}^{2} \times\left\{0_{\mathbf{C}_{p}}\right\} \times\right]-\beta_{k ;}^{\prime \prime}+\beta_{k}^{\prime \prime}\left[\stackrel{\phi_{k}^{\prime \prime}}{\longrightarrow} \mathbf{R}^{4+2 p}\right. \\
\left(x^{\prime}, x^{\prime \prime}, y^{\prime}, y^{\prime \prime}, 0, s_{k}^{\prime \prime}\right) \longmapsto \emptyset_{k}^{\prime \prime}\left(x^{\prime}, x^{\prime \prime}, y^{\prime}, y^{\prime \prime}, 0, s_{k}^{\prime \prime}\right)=\phi_{k, s_{k}^{\prime \prime} k}^{\prime \prime}\left(x^{\prime}, x^{\prime \prime}, y^{\prime}, y^{\prime \prime}, 0\right)
\end{gathered}
$$

où $\stackrel{\circ}{W}_{k}^{\prime}$ et $\stackrel{\circ}{W}_{k}^{\prime \prime}$ désignent respectivement l'intérieur de $W_{k}^{\prime}$ et $W_{k}^{\prime \prime}$. Les applications $\emptyset_{k}^{\prime}$ et $\emptyset_{k}^{\prime \prime}$ étant continues on peut trouver un voisinage ouvert $W$ de 0 dans $\mathbf{R}^{4+2 p}$ et un nombre réel $\varepsilon$ strictement positif assez petit telle que l'application $\emptyset_{p}^{\prime \prime} \circ \phi_{p}^{\prime} \circ \ldots \circ \phi_{2}^{\prime \prime} \circ \phi_{2}^{\prime} \circ \emptyset_{1}^{\prime \prime} \circ \emptyset_{1}^{\prime}$ soit définie sur:

$$
\left.W \cap \mathbf{C}^{2} \times\left\{0_{\mathbf{C} p}\right\} \times\right] \varepsilon,+\varepsilon[
$$

Posons:

$-V=W \cap C^{2} \times\{0\}$ 
- $\alpha_{k}^{\prime}=\inf \left(\varepsilon, \beta_{k}^{\prime}\right) \quad 1 \leq k \leq p$

- $\alpha_{k}^{\prime \prime}=\inf \left(\varepsilon, \beta_{k}^{\prime \prime}\right) \quad 1 \leq k \leq p$

Considérons l'application $\phi$ suivante de

$V \times]-\alpha_{1}^{\prime},+\alpha_{1}^{\prime},[\times]-\alpha_{1}^{\prime \prime},+\alpha_{1}^{\prime \prime},[\times]-\alpha_{2}^{\prime},+\alpha_{1}^{\prime},[\times]-\alpha_{2}^{\prime \prime},+\alpha_{2}^{\prime \prime}[\times \cdots \times] \alpha_{p}^{\prime},+\alpha_{p}^{\prime},[\times]-$ $\alpha_{p}^{\prime \prime},+\alpha_{p}^{\prime \prime}[$

dans $\mathbf{R}^{4+2 p} \simeq \mathbf{C}^{2} \times \mathbf{C}^{p}$ définie par:

1) si $\left(x^{\prime}, x^{\prime \prime}, y^{\prime}, y^{\prime \prime}\right) \neq(0,0,0,0)$ on pose:

$$
\begin{gathered}
\phi\left(x^{\prime}, x^{\prime \prime}, y^{\prime}, y^{\prime \prime}, s_{1}^{\prime}, s_{1}^{\prime \prime}, s_{2}^{\prime}, s_{2}^{\prime \prime}, \cdots, s_{p}^{\prime}, s_{p}^{\prime \prime}\right)= \\
=\left(\phi _ { p , s _ { p } ^ { \prime \prime } } ^ { \prime \prime } \circ \phi _ { p , s _ { p } ^ { \prime } } ^ { \prime } \circ \circ \circ \phi _ { 2 , s _ { 2 } ^ { \prime \prime } } ^ { \prime \prime } \circ \phi _ { 1 , s _ { 1 } ^ { \prime \prime } } ^ { \prime \prime } \circ \phi _ { 1 , s _ { 1 } ^ { \prime } } ^ { \prime } \left(x^{\prime}, x^{\prime \prime}, y^{\prime}, y^{\prime \prime}, 0,0, \cdots, 0, s_{1}^{\prime}, s_{1}^{\prime \prime},\right.\right. \\
\left.\left.s_{2}^{\prime}, s_{2}^{\prime \prime}, \cdots, s_{p}^{\prime}, s_{p}^{\prime \prime}\right)\right)
\end{gathered}
$$

2) si $\left(x^{\prime}, x^{\prime \prime}, y^{\prime}, y^{\prime \prime}\right)=(0,0,0,0)$ on pose:

$\phi\left(x^{\prime}, x^{\prime \prime}, y^{\prime}, y^{\prime \prime}, s_{1}^{\prime}, s_{1}^{\prime \prime}, s_{2}^{\prime}, s_{2}^{\prime \prime}, \cdots, s_{p}^{\prime}, s_{p}^{\prime \prime}\right)=\left(0,0,0,0, s_{1}^{\prime}, s_{1}^{\prime \prime}, s_{2}^{\prime}, s_{2}^{\prime \prime}, \cdots, s_{p}^{\prime}, s_{p}^{\prime \prime}\right)$.

On vérifie sans peine que $\phi$ est bien définie, injective, continue, ouverte et par conséquent $\phi$ est un homéomorphisme local de $\left(C^{2} \times C^{p}, 0\right)$.

$\phi$ convient car il commute avec la projection sur $C^{p}$, et suit les trajectoires des champs de vecteurs $\chi_{1}^{\prime}, \chi_{2}^{\prime}, \cdots, \chi_{p}^{\prime}, \chi_{1}^{\prime \prime} \chi_{2}^{\prime \prime}, \cdots, \chi_{p}^{\prime \prime}$. De plus $\phi$ transforme le produit par $C^{p}$ du feuilletage $\mathcal{F}_{\omega_{0}}$, défini par $\omega_{0}$, en le feuilletage $\mathcal{F}_{\omega}$ défini par $\omega:$ En effet, soit $F$ une feuille de $\mathcal{F}_{\omega}$ et désignons par $F_{0}$ la restriction de $F$ à $C^{2} \times\{0\}$. Montrons que:

$$
\phi\left(F_{0} \times\left(C^{p}, 0\right)\right)=F
$$

Pour $\left(m, s_{1}^{\prime}, s_{1}^{\prime \prime}, s_{2}^{\prime}, s_{2}^{\prime \prime}, \cdots, s_{p}^{\prime}, s_{p}^{\prime \prime}\right) \in F_{0} \times\left(C^{p}, 0\right)$ on a:

$\left.\phi_{p, s_{p}^{\prime \prime}}^{\prime \prime} \circ \phi_{p, s_{p}^{\prime}}^{\prime} \circ \cdots \circ \phi_{2, s_{2}^{\prime \prime}}^{\prime \prime} \circ \phi_{2, s_{2}^{\prime}}^{\prime} \circ \phi_{1, s_{1}^{\prime \prime}}^{\prime \prime} \circ \phi_{1, s_{1}^{\prime}}^{\prime}(m) \in F\right|_{C^{2} \times\left\{\left(s_{1}^{\prime}, s_{1}^{\prime \prime}, s_{2}^{\prime}, s_{2}^{\prime \prime}, \cdots, s_{p}^{\prime}, s_{p}^{\prime \prime}\right)\right\}}$.

(car les champs de vecteurs $\chi_{1}^{\prime}, \chi_{2}^{\prime}, \cdots, \chi_{p}^{\prime}, \chi_{1}^{\prime \prime} \chi_{2}^{\prime \prime}, \cdots, \chi_{p}^{\prime \prime}$ ) sont tangents au feuilletage $\left.\mathcal{F}_{\omega}\right)$.

Donc $\phi\left(m, s_{1}^{\prime}, s_{1}^{\prime \prime}, s_{2}^{\prime}, s_{2}^{\prime \prime}, \cdots, s_{p}^{\prime}, s_{p}^{\prime \prime}\right) \in F$.

Réciproquement, soit

$$
\left(x^{\prime}, x^{\prime \prime}, y^{\prime}, y^{\prime \prime}, s_{1}^{\prime}, s_{1}^{\prime \prime}, s_{2}^{\prime}, s_{2}^{\prime \prime}, \cdots, s_{p}^{\prime}, s_{p}^{\prime \prime}\right) \in F .
$$

et posons:

$$
\begin{gathered}
m=\phi_{1,-s_{1}^{\prime}}^{\prime} \circ \phi_{1,-s_{1}^{\prime \prime}}^{\prime \prime} \circ \phi_{2,-s_{1}^{\prime}}^{\prime} \circ \phi_{2,-s_{2}^{\prime \prime}}^{\prime \prime} \circ \cdots \circ \phi_{p,-s_{p}^{\prime}}^{\prime} \circ \\
\circ \phi_{p,-s_{p}^{\prime \prime}}^{\prime \prime}\left(x^{\prime}, x^{\prime \prime}, y^{\prime}, y^{\prime \prime}, s_{1}^{\prime}, s_{1}^{\prime \prime}, s_{2}^{\prime}, s_{2}^{\prime \prime}, \cdots, s_{p}^{\prime}, s_{p}^{\prime \prime}\right)
\end{gathered}
$$


On a: $m \in F_{0}$. De plus

$$
\phi\left(m, s_{1}^{\prime}, s_{1}^{\prime \prime}, s_{2}^{\prime}, s_{2}^{\prime \prime}, \cdots, s_{p}^{\prime}, s_{p}^{\prime \prime}\right)=\left(x^{\prime}, x^{\prime \prime}, y^{\prime}, y^{\prime \prime}, s_{1}^{\prime}, s_{1}^{\prime \prime}, s_{2}^{\prime}, s_{2}^{\prime \prime}, \cdots, s_{p}^{\prime}, s_{p}^{\prime \prime}\right)
$$

D'où:

$$
\phi\left(F_{0} \times\left(\mathbf{C}^{p}, 0\right)\right)=F
$$

de qui achève la démonstration.

\section{Trivialité analytique, type topologique constant.}

Dans tout ce qui suit nous désignons par $\omega$ un germe de formes de Pfaff holomorphes intégrables à l'origine 0 de $C^{n}=C^{2} \times C^{p}$ dont le lieu singulier $S$ est une sous-variété lisse de codimension 2, disons $S=\{(0,0)\} \times C^{p}$. Ecrivons:

$$
\omega(x, y, z)=a(x, y, z) d x+b(x, y, z) d y+\sum_{k=1}^{p} c_{k}(x, y, z) d z_{k}
$$

avec $(x, y) \in C^{2}$ et $z=\left(z_{1}, z_{2}, \cdots, z_{p}\right) \in C^{p}$ et désignons par $\omega_{z}$ la restriction de $\omega$ à $C^{2} \times\{z\}$ :

$$
\begin{aligned}
& \omega_{z}(x, y)=a(x, y, z) d x+b(x, y, z) d y \\
& \omega_{0}(x, y)=a(x, y, 0) d x+b(x, y, 0) d y
\end{aligned}
$$

Définition 3.1. Nous dirons que $\omega$ est analytiquement trivial au-dessus de $\omega_{0}$ s'il existe une unité $h \in \mathcal{O}_{C^{n}, 0}$ et un germe de difféomorphismes analytiques $\phi$ de $C^{n}=C^{2} \times C^{p}$ qui commute avec la projection sur $C^{p}$ et qui trivialise le germe de feuilletages $\mathcal{F}_{\omega}$ défini par $\omega$ :

$$
\begin{aligned}
& \phi^{*} \omega=h \cdot \omega_{0} \quad, \quad h(0) \neq 0 . \\
& (x, y, z) \in\left(C^{n}, 0\right) \quad \longrightarrow \quad \phi(x, y, z) \in\left(C^{n}, 0\right) \\
& z \in \mathbf{C}^{p} \\
& \swarrow \pi
\end{aligned}
$$

Nous avons le

Théorème 3.1. Si pour tout $k, 1 \leq k \leq p \quad c_{k} \in(a, b) \mathcal{O}_{C^{n, 0}}$ alors $\omega$ est analytiquement trivial au-dessus de $\omega_{0}$. 
$(a, b) \mathcal{O}_{C^{n}, 0}$ étant l'idéal, de l'anneau local $\mathcal{O}_{C^{n}, 0}$, engendré par $a$ et $b$.

Soit $k, 1 \leq k \leq p$. On a $c_{k} \in(a, b) \cdot \mathcal{O}_{C^{n}, 0}$, donc il existe $A_{k}, B_{k} \in \mathcal{O}_{C^{n}, 0}$ tels que:

$$
c_{k}=A_{k} a+B_{k} b
$$

Considérons le germe de champs de vecteur défini par:

$$
\chi=\left(-\sum_{k=1}^{p} A_{k}\right) \frac{\partial}{\partial x}+\left(-\sum_{k=1}^{p} B_{k}\right) \frac{\partial}{\partial y}+\frac{\partial}{\partial z_{1}}+\frac{\partial}{\partial z_{2}}+\cdots+\frac{\partial}{\partial z_{p}}
$$

on a : $\omega(\chi) \equiv 0$. De plus, en intégrant $\chi$ on trouve le germe de difféomorphisme $\Phi$ cherché.

Définition 3.2. Nous dirons que $\omega$ est à type topologique constant si pour tout $z$ voisin de 0 il existe un germe d'homéomorphismes $\phi_{2}:\left(C^{2} \times\right.$ $\{z\}, 0) \longrightarrow\left(C^{2} \times\{0\}, 0\right)$ qui transforme le germe de feuilletages défini par $\omega_{z}$ en le germe de feuilletages défini par $\omega_{0}$.

Remarque 3.1. Le notion de type topologique constant n'est pas équivalente à la notion de trivialité topologique. Il est clair que "trivialité topologique" implique "type topologique constant" mais la réciproque est fausse pour s'en convaincre nous renvoyons le lecteur à [4] où nous avons étudié de façon détaillée l'exemple suivant:

$$
\omega(x, y, z)=\left(2 y^{2}+x^{3}\right) d x-2 x y d y+\sum_{k=1}^{p} x^{3} d z_{k}
$$

Nous avons la:

Proposition 3.1. Si la condition $\left(c_{\omega}\right)$ est vérifiée le long de $S$ alors $\omega$ est à type topologique constant.

D'après le lemme d'isotopie, il existe un germe d'homéomorphismes $\phi$ de $\left(C^{n}, 0\right)$ qui commute avec la projection et qui transforme le germe de feuilletages défini par $\omega$ en le produit par $C^{p}$ du germe de feuilletages défini par $\omega_{0}$.

Soit $\phi_{z}$ l'application définie par:

$$
\phi_{z}(x, y)=\phi(x, y, z)
$$

$\phi_{z}$ est l'homéomorphisme cherché. 


\section{4. Écriture algébrique des conditions $\left(a_{\omega}\right)$ et $\left(c_{\omega}\right)$}

Rappelons que si $\varphi$ est un germe de fonctions holomorphes à l'origine 0 de $C^{n}$ et $\gamma:\left(C^{n}, 0\right)$ un germe de chemins analytiques de $C^{n}$ en 0 , on appelle ordre de $\varphi$ en 0 le long de $\gamma$ et on note $\nu_{\gamma}(\varphi)$ l'ordre usuel $\nu(\varphi \circ \gamma)$ de $\varphi \circ \gamma$ en $0: \nu_{\gamma}(\varphi)=\nu(\varphi \circ \gamma)$.

Plus généralement, soit $I=\left(\varphi_{1}, \varphi_{2}, \cdots, \varphi_{k}\right) \cdot \mathcal{O}_{C^{n}, 0}$ un idéal de $\mathcal{O}_{C^{n}, 0}$ engendré par $\varphi_{1}, \varphi_{2}, \cdots, \varphi_{k}$, et soit $\gamma:(C, 0) \longrightarrow\left(C^{n}, 0\right)$ un germe de chemins analytiques de $C^{n}$ en 0 . On appelle ordre de $I$ le long de $\gamma$ et on note $\nu_{\gamma}(I)$ le nombre suivant:

$$
\nu_{\gamma}(I)=\inf \left\{\nu_{\gamma}\left(\varphi_{j}\right) / 1 \leq j \leq k\right\} .
$$

Remarque 4.1. $\nu_{\gamma}(I)$ est indépendant des générateurs de I. En effet:

$$
\nu_{\gamma}(I)=\nu\left(\gamma^{*}(I)\right) \text { avec } \gamma^{*}(I)=\{f \circ \gamma / f \in I\} .
$$

$\mathcal{O}_{C, 0}$ étant un anneau principal, il existe $h \in \mathcal{O}_{C, 0}$ tel que:

$$
\gamma^{*}(I)=(h) \cdot \mathcal{O}_{C, 0} \quad \text { et } \quad \nu_{\gamma}(I)=\nu(h) .
$$

Nous avons besoin de la notion de dépendance intégrale, nous nous contenterons de donner uniquement la définition. Pour plus de détails concernant cette notion, nous renvoyons le lecteur à [3], [6], [11].

Définition 4.1. On appelle clôture intégrale d'un idéal $I$ de $\mathcal{O}_{C^{n}, 0}$ et on note $\bar{I}$ l'idéal de $\mathcal{O}_{C^{n}, 0}$ formé des éléments $h$ qui vérifient une relation du type.

$$
h^{k}+a_{1} h^{k-1}+\cdots+a_{k}=0 \text { où } a_{i} \in I^{i} \quad 1 \leq i \leq k
$$

Soit maintenant $\omega=a(x, y, z) d x+b(x, y, z) d y+\sum_{k=1}^{p} c_{k}(x, y, z) d z_{k}$ un germe de formes de Pfaff holomorphes intégrables à l'origine 0 de $C^{n}=$ $C^{2} \times C^{p},(x, y,) \in C^{2}, z=\left(z_{1}, \cdots, z_{p}\right) \in C^{p}$, dont le lieu singulier est une sous-variété lisse de codimension 2 , disons $S=\{(0,0)\} \times C^{p}$. Comme pour les germes de fonctions nous avons les résultats suivants:

Proposition 4.1. Les conditions suivantes sont équivalentes:

i) $\left(a_{\omega}\right)$ est vérifiée relativement à $S$

ii) Pour tout $k, 1 \leq k \leq p: \frac{c_{k}}{\sqrt{|a|^{2}+|b|^{2}}} \longrightarrow 0$ le long de $S$.

iii) Pour tout germe de chemins analytiques $\gamma:(C, 0) \longrightarrow\left(C^{n}, 0\right)$ et pour tout $k, 1 \leq k \leq p$ on a:

$$
\nu_{\gamma}\left(c_{k}\right)>\nu_{\gamma}\left((a, b) \cdot \mathcal{O}_{C^{n}, 0}\right)
$$


en exercice ou cf. [4].

Proposition 4.2. Les conditions suivantes sont équivalentes:

i) $\left(c_{\omega}\right)$ est vérifiée relativement à $S$

ii) il existe une constante $K>0$ et un voisinage ouvert $V$ de 0 dans $C^{n}$ tels que pour tout $k, 1 \leq k \leq p$ :

$$
\frac{\left|c_{k}\right|}{\sqrt{|a|^{2}+|b|^{2}}} \leq K \sqrt{|x|^{2}+|y|^{2}} \text { pour }(x, y, z) \in V
$$

iii) Pour tout $k, 1 \leq k \leq p: c_{k} \in \overline{(x, y)(a, b) \cdot \mathcal{O}_{C^{n}, 0}}$

iv) Pour tout germe de chemins analytiques $\gamma:(C, 0) \longrightarrow\left(C^{n}, 0\right)$ et pour tout $k, 1 \leq k \leq p$, on a:

$$
\nu_{\gamma}\left(c_{k}\right) \geq \nu_{\gamma}\left((x, y)(a, b) \cdot \mathcal{O}_{C^{n}, 0}\right)
$$

Elle découle sans peine de la proposition 3 du $§ 2$ et des deux lemmes suivants:

Lemme 4.1. (cf [3],[6]). Soit $I=\left(\varphi_{1}, \varphi_{2}, \cdots, \varphi_{h}\right) \cdot \mathcal{O}_{C^{n}, 0}$, un idéal de $\mathcal{O}_{C^{n}, 0}$ engendré $\operatorname{par} \varphi_{1}, \varphi_{2}, \cdots, \varphi_{h}$ et soit $\psi \in \mathcal{O}_{C^{n}, 0}$, on a alors : $\psi \in \bar{I}$ si et seulement si, il existe un voisinage ouvert $U$ de 0 dans $C^{n}$ et une constante $\lambda \in \mathbf{R}_{11}$ tels que:

$$
|\psi(m)| \leq \lambda \sup _{1 \leq j \leq h}\left|\varphi_{j}(m)\right| \text { pour tout } m \in U .
$$

Lemme 4.2. (cf. [11]). Soit $I$ un idéal de $\mathcal{O}_{C^{n}, 0}$ et $h \in \mathcal{O}_{C^{n}, 0}$ alors on a l'équivalence suivante: $h \in \bar{I}$ si et seulement si pour tout germe de chemins analytique $\gamma:(C, 0) \longrightarrow\left(C^{n}, 0\right)$ on a:

$$
\nu_{\gamma}(h) \geq \nu_{\gamma}(I)
$$

\section{Conditions $\left(a_{\omega}\right),\left(c_{\omega}\right)$ et $\mu$ constant.}

Rappelons que l'on appelle nombre de Milnor (cf. [10]) d'un germe $f$ de fonctions holomorphes à l'origine 0 de $C^{n}$, à singlularité isolée en 0 et l'on note $\mu(f)$ le nombre suivant:

$$
\mu(f)=\operatorname{dim}_{C}\left[\mathcal{O}_{C^{n}, 0} / J(f)\right]
$$


où $J(f)$ désigne l'idéal jacobien de $f$.

Cette notion porte uniquement sur $d f$ et non sur $f$ d'où la définition suivante:

Définition 5.1. On appelle multiplicité d'intersection en 0 , d'un germe de formes de $P$ faff $\Omega$ holomorphes en $0 \in C^{n}$, a singularité isolée en 0 et on note $\mu(\Omega)$ le nombre suivant:

$$
\mu(\Omega)=\operatorname{dim}_{C}\left(\mathcal{O}_{C^{n}, 0} / j(\Omega)\right),
$$

où $J(\Omega)$ désigne l'idéal de $\mathcal{O}_{C^{n}, 0}$ engendré par les composantes de $\Omega$.

Remarque 5.1. Si $\Omega=d f$ on retrouve la notion de nombre de Milnor d'un germe de fonctions.

Soit $\omega=a(x, y, z) d x+b(x, y, z) d y+\sum_{k=1}^{p} c_{k}(x, y, z) d z_{k}$ un germe de formes de Pfaff holomorphes intégrables à l'origine 0 de $C^{n}=C^{2} \times C^{p}$ dont le lieu singulier $S$ est égal à l'axe des $z, z=\left(z_{1}, z_{2}, \cdots, z_{p}\right) \in C^{p}$. Notons:

$$
\omega_{z}=\omega_{\mid C^{2} \times\{z\}}=a(x, y, z) d x+b(x, y, z) d y
$$

et désignons par $\mu_{z}$ la multiplicité d'intersection $\mu\left(\omega_{z}\right)$ de $\omega_{z}$ dans $\mathcal{O}_{C^{2}, 0}$. Comme pour les germes de fonctions, nous avons:

Proposition 5.1. $\left(a_{\omega}\right) \Longrightarrow \mu_{z}$ constant.

i.e., si la condition $\left(a_{\omega}\right)$ est vérifiée relativement à $S$ alors $\mu_{z}$ est indépendant de $z$.

Nous avons $S=\left\{a=b=c_{1}=c_{2}=\cdots c_{p}=0\right\}$. Pour montrer que $\mu_{z}$ est indépendant de $z$ il est équivalent de montrer que $\{a=b=0\}$ est égal à l'axe des $z$.

Comme par hypothèse $S$ est l'axe des $z$, il suffit de vérifier l'inclusion $\{a=b=0\} \subset S$.

La condition $\left(a_{\omega}\right)$ étant vérifiée relativement à $S$, il existe un voisinage ouvert $U$ de 0 dans $C^{n}$ tel que pour tout $k, 1 \leq k \leq p$ :

$$
\left|c_{k}(x, y, z)\right| \leq \sqrt{|a(x, y, z)|^{2}+|b(x, y, z)|^{2}} \text { pour }(x, y, z) \text { dans } U .
$$

Soit donc $(x, y, z) \in\{a=b=0\}$, comme:

$$
\left|c_{k}(x, y, z)\right| \leq \sqrt{|a(x, y, z)|^{2}+|b(x, y, z)|^{2}} .
$$

Nous avons $c_{k}(x, y, z)=0$ pour tout $k, 1 \leq k \leq p$ donc $(x, y, z) \in S$. Et la proposition est démontrée. 
Corollaire 5.1. $\left(c_{\omega}\right) \Longrightarrow \mu_{z}$ constant.

i.e., si la condition $\left(c_{\omega}\right)$ est vérifiée relativement à $S$ alors $\mu_{z}$ est indépendant de $z$.

Remarque 5.2. La réciproque de la proposition précédente est fausse. En effet, considérons le germe de Pfaff $\omega$ à l'origine de $C^{n}$ défini par:

$$
\omega(x, y, z)=2 y^{2} d x-2 x y d y+\sum_{k=1}^{p} x^{3} d z_{k}
$$

où $(x, y) \in C^{2}$ et $z=\left(z_{1}, z_{2}, \cdots, z_{p}\right) \in C^{p}$ avec $C^{n}=C^{2} \times C^{p}$.

On vérifie sans peine que $\omega$ est intégrable, son lieu singulier $S$ est égal à l'axe des $z, \mu_{z}$ est indépendant de $z$ mais la condition $\left(c_{\omega}\right)$ n'est vérifiée en aucun point de $S$ relativement à $S$.

\section{Récapitulatif.}

Soit $\omega$ un germe de formes de Pfaff holomorphes intégrables à l'origine $0 \in C^{n}$ dont le lieu singulier $S$ est lisse de codimension 2. Nous avons le diagramme suivant:

$$
\begin{gathered}
\left(a_{w}\right) \Longleftarrow\left(c_{w}\right) \Longrightarrow(w t \cdot t) \\
\left.\bigcup \|\left(\left(w_{z}\right)_{z}\right) t \cdot t \cdot c\right)
\end{gathered}
$$

$\left(a_{\omega}\right)$ : la condition $\left(a_{\omega}\right)$ est vérifiée relativement à $S$. $\left(c_{\omega}\right):$ la condition $\left(c_{\omega}\right)$ est vérifiée relativement à $S$. $\omega t \cdot t: \omega$ est topologiquement trivial.

$\left.\left(\left(\omega_{z}\right)_{z}\right) t \cdot t \cdot c\right)$ : la famille $\left(\omega_{z}\right)_{z}$ est à type topologique constant. $\left(\mu_{z} c\right): \mu_{z}$ est indépendant de $z$. 


\section{References}

[1] J. Briançon et J-P. Speder:Les conditions de Whitney impliquant $\mu^{*}$ constant. Annales de l'Institut fourier de Grenoble, 26 (2), p. 153-163 (1976).

[2] Camacho, C.; Lins Neto, A. et Sad, P.: Topological invariants and equidesingularization for holomorphic vector fields, Journal of differential Geometry, 20, p. 143-174 (1984).

[3] Hironaka, H.: Introduction to the Theory of infinitely near singular points Memorias de Matemática del Instituto "Jorge Juan" 28 Madrid (1974).

[4] Kabila, A.: Formes intégrables lieu singulier lisse: Conditions de Whitney. Equisingularit. $\mu$ constant. Thèse de 3me cycle, Dijon (1983).

[5] Kabila, A.: Formes intégrables lieu singulier lisse: CRAS Tome 298, Série I $n^{\circ} 11$ (1984).

[6] Kupka, I.: Singularities of integral structuraly stable Pfaffian forms. Proc. Nat. Sciences, 52 (1964).

[7] Lé Dung Tráng: Calcul du nombre de cycles évanouissants d'une hypersurface complexe. Ann.Inst. Fourier, Grenoble, 23,4, 261-270 (1973).

[8] M. Lejeune-Jalabert, M. et Teissier, B.: Fermeture intégrable des idéaux et équisingularité. Séminaire Ecole Polytechnique 1973-74 Centre de Math. 91128 Palaiseau Cedex.

[9] B. Malgrange, B.: Frobenius avec singularité 1: Codimension un Pub. Math. I.H.E.S. 46, 163-173 (1976).

[10] J. Milnor: Singular points of complexe hypersurfaces. Annals of Mathematics Studies - Princeton Univ. Press.

[11] J-P. Speder: Equisingularité et conditions de Withney Thése d'Etat, Université de Nice (1976). 
[12] Teissier, B.: The hunting of invariants in geometry of discriminants. Ecole Polytechnique - Centre de Mathématiques - 91128 Palaiseau Cedex.

[13] Teissier, B.: Cycles evanescents, sections planes et conditions de Whitney. Singularités à Cargèse - Astéristique 7 et 8 (1973).

[14] Teissier, B.: Introduction to equisingularity problems. Proceding of symposia in Pure Mathematics Vol, 29, p. 593-632 (1975).

[15] R. Thom: Ensembles et morphismes stratifiés. Bull. Amer. Math. Soc. 75 (1969).

Received: 20 July 1994
Abdelhak Kabila
URA au CNRS D.751
Département de Mathématiques
Université de Valenciennes et du Hainaut Cambrésis
I.S.T.V., B.P. 311
59304 Valenciennes Cedex 